\title{
EDITORIAL
}

\section{Roadmaps in a nutshell}

\author{
This month we publish our first Roadmap. Here is what readers, authors and referees need to \\ know about this article type.
}

In this issue, we publish a Roadmap by Brant Robertson and colleagues on how large astronomical surveys can help understand galaxy formation. We highly recommend you read it, whether you work in this research field or not. It is the first Roadmap-type article in Nature Reviews Physics and it illustrates what we are aiming for with such content.

Roadmaps are by no means new, but they are becoming more popular, which perhaps reflects the changing needs of the research community. Recent examples that spring to mind are the well-known 2018 quantum technologies roadmap ${ }^{1}$ or the 2017 magnetism roadmap ${ }^{2}$. These articles briefly overview the current state-of-the-art in the field and discuss the challenges and opportunities. As such they are accessible and extremely valuable to young researchers entering a new field, or scientists interested in shifting to a new area or looking for new collaboration opportunities. Recognizing the great value of this type of resource, we created the Roadmap article type in Nature Reviews Physics. Here is what you can expect from it.

As a reader, a Roadmap article should provide you with a broad overview of the field, a sense of where the field is going and what the open questions are. As a graduate student or junior researcher, the bigger picture might help you understand where your own research fits in a broader context. Roadmaps are meant to foster interdisciplinary collaborations and knowledge transfer, because they are more accessible and concise, compared

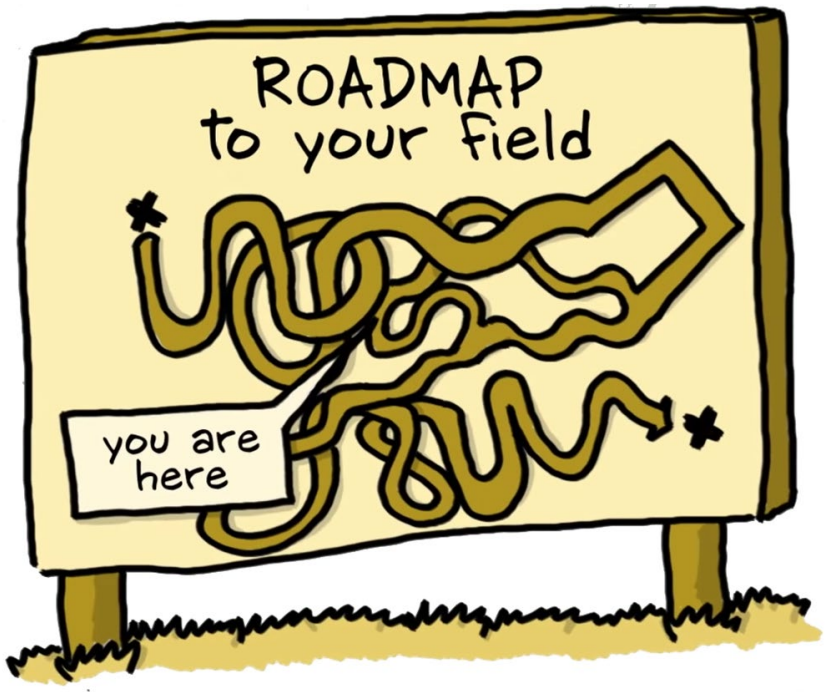

with the large volumes of technical literature, and because they emphasize current key questions and approaches to addressing them.

As an author, the Roadmap format allows you to do more than just survey the literature. It allows you to write about issues that are not explicitly mentioned in research papers, but much talked about in the community (for example, concerning device characterization or reproducibility). As with Reviews, Roadmaps should be balanced and reflect the views of the consensus in the field. They should be written for scientists, not for funders, and therefore timelines, objective-setting and planning are not necessarily relevant (despite what the name may suggest).

As a referee, an important thing to keep in mind is that Roadmaps are not supposed to be exhaustive overviews of the literature, but rather brief recaps of the current status of research followed by more extensive forward-looking discussions. As you are an expert in the field, some things may seem obvious, and you need to think of the broader readership the article is intended for. Good questions to ask yourself about the Roadmap article you are refereeing are does it reflect the views of the community; does it explain the challenges and opportunities clearly and succinctly; is it timely, balanced and accurate; and is it accessible to a graduate physicist from a different area of physics?

Roadmap articles are naturally suited for long-term projects, large instruments and applied research, with which goals are clearly defined. But this does not mean that this article type is restricted to such topics. Developments in science cannot be predicted and the most interesting results are the unexpected ones. So there is a risk that Roadmaps may become outdated very quickly if an unexpected discovery is made, solving or making obsolete certain issues. But the majority of Roadmaps are likely to provide a path to discovery and progress for years to come.

Whether a field is growing quickly or moving slowly, whether it is theoretical or experimental, whether it involves many small groups or a few large instruments, it is for each community to find the best way of making use of Roadmaps to answer its own needs. We offer the format, guidance and support, and together with our authors, readers and referees we will explore the full potential of Roadmaps to serve the physics community.

Acin, A. et al. The quantum technologies roadmap: a European community view. New J. Phys. 20, 080201 (2018).

2. Sander, D. et al. The 2017 magnetism roadmap. J. Phys. D: Appl. Phys. 50, 363001 (2017) 